\title{
Pesticide Options for Insect, Mite, and Mollusk Management in Commercial Strawberry Production in Florida ${ }^{1}$
}

\author{
James F. Price, Curtis Nagle, and Susan E. Webb²
}

Florida growers produce primarily fresh market strawberries that were valued at $\$ 366.3$ million in $2010-11$, harvested from 9,900 acres (Florida Agriculture Statistical Bulletin 2011). More than $95 \%$ of the crop is produced near Plant City, with smaller production areas in north Florida and around Homestead, FL.

Major early-season arthropod pests include lepidopterous larvae, twospotted spider mites, and aphids, some of which may accompany the transplants from their origin. By mid-season and later, major concerns are with twospotted spider mites, thrips, fruit (vinegar) flies and sap beetles. Pameras (seed bugs) add to the concern and may evoke complaints when they accompany berries to markets. Now spotted wing drosophila flies can be present to damage fruit or to reproduce and damage the blueberry crop that follows strawberries. Widow spiders sometimes are problems in fields where broad spectrum insecticides are infrequently used.

Effective management of arthropod and gastropod pests of strawberry is critical to the profitability of the industry and requires that pests be detected in a timely manner through systematic scouting. Appropriate control measures should be applied as conditions warrant.

Biological control measures have been developed for management of twospotted spider mites and are practiced by a portion of the industry. Information on biological control of insects and mites in strawberry production is available at http://edis.ifas.ufl.edu/HS180. Toxicity information for many pesticides used in Florida strawberry production to commercially available predators of spider mites is summarized at http://side-effects.koppert.nl/.

The tables in this document list pesticides that are presently available to commercial strawberry producers in Florida and are organized alphabetically by the following major pest groups:

Ants

Aphids

Armyworms

1. This document is ENY-689 (IN486), one of a series of the Entomology \& Nematology Department, Florida Cooperative Extension Service, Institute of Food and Agricultural Sciences, University of Florida. Published: December 2003. Revised: December 2007 and April 2013 For more publications related to horticulture/agriculture, please visit the EDIS website at http://edis.ifas.ufl.edu.

2. James F. Price, associate professor, retired, Gulf Coast Resource and Education Center, Wimauma, 33598; Curtis Nagle, biological scientist, Entomology and Nematology Department, Gulf Coast Research and Education Center, Wimauma, 33598; and Susan E. Webb, associate professor, Entomology and Nematology Department, Cooperative Extension Service, Insititute of Food and Agricultural Sciences, Gainesville, 32611.

The use of trade names in this publication is solely for the purpose of providing specific information. UF/IFAS does not guarantee or warranty the products named, and references to them in this publication do not signify our approval to the exclusion of other products of suitable composition. All chemicals should be used in accordance with directions on the manufacturer's label. Use pesticides safely. Read and follow directions on the manufacturer's label. 
Beetles and weevils

Caterpillars (including budworms, earworms, leafrollers, leaftiers, lesser cornstalk borer and loopers)

Crickets

Fruit flies (vinegar flies) and spotted wing drosophila

Grubs

Mites

Mole crickets

Pameras

Plant (Lygus) bugs

Snails and slugs

Spiders

Thrips

Available pesticides for strawberry include beneficial nematode and microbial insecticides, which are components of biological control. For each pest group listed, products available for control are presented by the active ingredient's common name. Usually only one or a few examples of each formulation are given; however, there may be other products as effective as those listed. Notes taken from labels are provided to qualify some uses. More information about pesticide products can be found on electronic versions of specimen labels which are usually available at the websites of CDMS (http://www.cdms.net/manuf/default.asp), C\&P Press (http://www.greenbook.net/) or the affiliated manufacturer. The product label communicates the lawful use of the product and must be read, understood and followed. A label may contain important limitations that are not presented here, and it remains the pesticide applicator's legal responsibility to read and follow all label instructions on the container of the specific pesticide being used.

Many pesticides decompose in the spray tank when mixed with water above $\mathrm{pH} 7$. Growers should test the $\mathrm{pH}$ of their water and, when above 7 , should add a buffering solution to maintain $\mathrm{pH}$ between 6.5 and 7 . When using a pesticide for the first time, it is important to test the product first on a small portion of the crop and check for any possible detrimental effects over time, such as leaf distortion and plant stunting.

This summary is only a guide to aid in the proper selection of pesticides. Care has been given to provide accurate and up-to-date information, but it is possible that, through label changes, error, etc., improper uses may be indicated.
As an additional precaution, keep the telephone number and address of the nearest county poison control center in a convenient location in case of an accidental poisoning. Also, keep clean copies of labels of all pesticides on the farm premises. In the event of a poisoning, the label of the pesticide involved should be taken to the poison control center or hospital.

\section{Reference}

Florida Agriculture Statistical Bulletin 2011. 2012. http://www.nass.usda.gov/Statistics_by_State/Florida/ Publications/Annual_Statistical_Bulletin/fasb12/E1thru18Veg-2012.pdf, NASS, USDA. 
Table 1. Ant pesticidal control measures available for commercial strawberry production in Florida.

\begin{tabular}{|c|c|c|c|}
\hline Common Name & $\begin{array}{l}\text { Trade Name/ } \\
\text { Formulation }\end{array}$ & $\begin{array}{l}\text { Production } \\
\text { Site }^{1}\end{array}$ & Notes from labels ${ }^{2}$ \\
\hline $\begin{array}{l}\text { Beauveria bassiana } \\
\text { ATCC } 74040\end{array}$ & Naturalis L & $\mathrm{F}, \mathrm{G}, \mathrm{N}$ & $\begin{array}{l}\text { At least 3-5 applications may be necessary before pests are adequately } \\
\text { under control. Do not tank mix with fungicides. Wait a minimum of } 48 \\
\text { hours after application before applying fungicides. }\end{array}$ \\
\hline Carbaryl & $\begin{array}{l}10 \% \text { Sevin Granules } \\
\text { Cutworm \& Cricket } \\
\text { Bait }\end{array}$ & $\mathrm{F}, \mathrm{N}$ & $\begin{array}{l}\text { Repeat applications up to a total of four times per year, but not more } \\
\text { often than once every } 7 \text { days. Long, 7-day pre-harvest interval (PHI). }\end{array}$ \\
\hline $\begin{array}{l}\text { Methyl bromide \& } \\
\text { Chloropicrin }\end{array}$ & $\mathrm{MBC} 98-2^{3}$ & $\mathrm{~F}, \mathrm{G}, \mathrm{N}$ & Pre-plant treatment only. \\
\hline Pyrethrin & $\begin{array}{l}\text { PyGanic Crop } \\
\text { Protection } \\
\text { EC } 1.4^{4} \\
\text { PyGanic EC } 5.0^{4}\end{array}$ & $\mathrm{~F}, \mathrm{G}, \mathrm{N}$ & \\
\hline $\begin{array}{l}\text { Pyrethrin \& piperonyl } \\
\text { butoxide }\end{array}$ & $\begin{array}{l}\text { Evergreen EC } 60-6 \\
\text { Pyreth-It } \\
\text { Pyrenone Crop Spray }\end{array}$ & $\mathrm{F}, \mathrm{G}, \mathrm{N}$ & \\
\hline Pyriproxyfen & Esteem Ant Bait & $\mathrm{F}, \mathrm{G}, \mathrm{N}$ & $\begin{array}{l}\text { For } 7 \text { to } 10 \text { days after treatment, do not apply any other fire ant } \\
\text { pesticide. Do not exceed more than } 0.134 \mathrm{lb} \text {. of pyriproxyfen per acre } \\
\text { per season. }\end{array}$ \\
\hline S-methoprene & $\begin{array}{l}\text { Extinguish Pro Fire } \\
\text { Ant Bait }\end{array}$ & $\mathrm{F}, \mathrm{G}, \mathrm{N}$ & Fire ants. \\
\hline \multicolumn{4}{|c|}{$\begin{array}{l}\text { Footnotes: } \\
1 \text { "F" indicates field production. “G" indicates greenhouse production. “N" indicates nursery production. } \\
{ }^{2} \text { Notes are taken from product labels and restrict use to the condition indicated (suppression, beet armyworm, exposed thrips, etc.), limit } \\
\text { number and patterns of applications, provide phytotoxicity precautions, etc. } \\
{ }^{3} \text { Product is a restricted-use pesticide. } \\
{ }^{4} \text { Product label indicates use in organic production. }\end{array}$} \\
\hline
\end{tabular}


Table 2. Aphid pesticidal control measures available for commercial strawberry production in Florida.

\begin{tabular}{|c|c|c|c|}
\hline Common Name & Trade Name/ Formulation & Production Site $^{1}$ & Notes from labels ${ }^{2}$ \\
\hline Abamectin & Temprano $^{3}$ & $\mathrm{~F}$ & Suppression. \\
\hline Acetamiprid & Assail 30SG & $\mathrm{F}, \mathrm{G}, \mathrm{N}$ & $\begin{array}{l}\text { Do not make more than two applications per } \\
\text { growing season. }\end{array}$ \\
\hline Azadirachtin & $\begin{array}{l}\text { Aza-Direct }^{4} \\
\text { Azatin XL } \\
\text { Neemix } 4.5^{4} \\
\text { Ecozin Plus } 1.2 \% \mathrm{ME}^{4}\end{array}$ & $\mathrm{~F}, \mathrm{G}, \mathrm{N}$ & $\begin{array}{l}\text { Reduces damage by repelling and deterring } \\
\text { feeding of all stages of insect. }\end{array}$ \\
\hline Beauveria bassiana & $\begin{array}{l}\text { Naturalis L } \\
\text { BotaniGard ES } \\
\text { BotaniGard } 22 \text { WP } \\
\text { Mycotrol } \mathrm{O}^{4}\end{array}$ & $\mathrm{~F}, \mathrm{G}, \mathrm{N}$ & $\begin{array}{l}\text { Typically it takes } 7-10 \text { days after the first spray } \\
\text { to see control. At least } 3-5 \text { applications may be } \\
\text { necessary before pests are adequately under } \\
\text { control. See labels for precautions about use } \\
\text { with fungicides. }\end{array}$ \\
\hline Bifenthrin & Brigade WSB ${ }^{3}$ & $\mathrm{~F}, \mathrm{~N}$ & $\begin{array}{l}\text { Plant back restrictions: Plants for which } \\
\text { bifenthrin tolerances exist may be rotated at } \\
\text { any time. All other crops may be rotated } 30 \text { days } \\
\text { after the final application of bifenthrin. }\end{array}$ \\
\hline $\begin{array}{l}\text { Bifenthrin \& avermectin } \\
\text { B1 }\end{array}$ & Athena $^{3}$ & $\mathrm{~F}$ & $\begin{array}{l}\text { Do not make more than two consecutive } \\
\text { applications and four applications per growing } \\
\text { season. Plant back restrictions: For crops that } \\
\text { have bifenthrin and avermectin tolerances, the } \\
\text { plant back is immediate. All other crops may be } \\
\text { rotated } 30 \text { days after the final application. }\end{array}$ \\
\hline Bifenthrin \& imidacloprid & Brigadier $^{3}$ & $\mathrm{~F}, \mathrm{~N}$ & $\begin{array}{l}\text { Do not apply during or within } 10 \text { days after } \\
\text { bloom or when bees are actively foraging. Long, } \\
\text { 7-day pre-harvest interval (PHI). Plant back } \\
\text { restrictions: Plants that have tolerances for both } \\
\text { bifenthrin and imidacloprid may be rotated } \\
\text { at any time. Crops that have tolerances for } \\
\text { bifenthrin and not imidacloprid can be rotated } \\
12 \text { months after the final application of Brigadier } \\
\text { insecticide. Crops that have tolerances for } \\
\text { imidacloprid and not bifenthrin may be rotated } \\
30 \text { days after the final application [label has list } \\
\text { of crops]. }\end{array}$ \\
\hline Diazinon & $\begin{array}{l}\text { Diazinon } \mathrm{AG} 500^{3} \\
\text { Diazinon } 50 \mathrm{~W}^{3} \\
\text { Diazinon } \mathrm{AG} 600 \mathrm{WBC}^{3}\end{array}$ & $\mathrm{~F}, \mathrm{~N}$ & $\begin{array}{l}\text { Make a maximum of one foliar application per } \\
\text { crop and a maximum of one soil application per } \\
\text { crop. Long, 5-day pre-harvest interval (PHI). }\end{array}$ \\
\hline $\begin{array}{l}\text { Edible fish oil \& sesame } \\
\text { oil }\end{array}$ & Organocide 3-in-1 Garden Spray ${ }^{4}$ & $\mathrm{~F}, \mathrm{G}, \mathrm{N}$ & \\
\hline Flonicamid & Beleaf 50 SG & $\mathrm{F}, \mathrm{N}$ & \\
\hline Imidacloprid & $\begin{array}{l}\text { Admire Pro } \\
\text { Couraze } 2 \mathrm{~F} \\
\text { Provado } 1.6 \text { Flowable }\end{array}$ & $\mathrm{F}, \mathrm{N}$ & $\begin{array}{l}\text { Do not apply during bloom or within } 10 \\
\text { days before bloom or when bees are actively } \\
\text { foraging. }\end{array}$ \\
\hline $\begin{array}{l}\text { Isaria fumosorosea } \\
\text { (formerly Paecilomyces } \\
\text { fumosoroseus) }\end{array}$ & $\begin{array}{l}\text { Preferal } \\
\text { PFR-97 20\% WDG }{ }^{4}\end{array}$ & $\mathrm{~F}, \mathrm{G}, \mathrm{N}$ & $\begin{array}{l}\text { Most effective when relative humidity is } 80 \% \\
\text { or higher for } 8-10 \text { hours. Can be mixed with } \\
\text { copper-based fungicides; do not mix with other } \\
\text { fungicides or apply within } 5 \text { days of fungicide } \\
\text { applications other than copper. }\end{array}$ \\
\hline Malathion & $\begin{array}{l}\text { Gowan Malathion } 8 \mathrm{~F} \\
\text { Malathion 5EC }\end{array}$ & $\mathrm{F}, \mathrm{N}$ & \\
\hline Naled & Dibrom 8 Emulsive ${ }^{3}$ & $\mathrm{~F}, \mathrm{~N}$ & Do not apply when temperature is over $90^{\circ} \mathrm{F}$. \\
\hline Neem oil & Trilogy & $\mathrm{F}, \mathrm{N}$ & $\begin{array}{l}\text { Avoid tank mixes with captan, sulfur or } \\
\text { other chemically similar products because } \\
\text { unpredictable results or leaf burn may occur. }\end{array}$ \\
\hline
\end{tabular}




\begin{tabular}{|c|c|c|c|}
\hline Common Name & Trade Name/ Formulation & Production Site ${ }^{1}$ & Notes from labels ${ }^{2}$ \\
\hline $\begin{array}{l}\text { Oil (mineral, paraffinic, } \\
\text { petroleum, vegetable, } \\
\text { etc.) }\end{array}$ & $\begin{array}{l}\text { Saf-T-Side Spray Oil } \\
\text { Ultra-Fine Oil }\end{array}$ & $\mathrm{F}, \mathrm{N}$ & See labels for phytotoxicity precautions. \\
\hline $\begin{array}{l}\text { Potassium Salts of Fatty } \\
\text { Acids } \\
\text { (insecticidal soap) }\end{array}$ & $\begin{array}{l}\text { AllPro Insecticidal Soap } 40 \% \\
\text { M-Pede }\end{array}$ & $\mathrm{F}, \mathrm{G}, \mathrm{N}$ & $\begin{array}{l}\text { Do not mix with sulfur. See labels for } \\
\text { phytotoxicity precautions. }\end{array}$ \\
\hline Potassium silicate & Sil-MATRIX & $\mathrm{F}, \mathrm{G}, \mathrm{N}$ & Suppression. \\
\hline Pyrethrin & $\begin{array}{l}\text { PyGanic Crop Protection EC } 1.4^{4} \\
\text { PyGanic EC } 5.0^{4}\end{array}$ & $\mathrm{~F}, \mathrm{G}, \mathrm{N}$ & \\
\hline $\begin{array}{l}\text { Pyrethrin \& piperonyl } \\
\text { butoxide }\end{array}$ & $\begin{array}{l}\text { EverGreen EC 60-6 } \\
\text { Pyreth-It } \\
\text { Pyrenone Crop Spray }\end{array}$ & $\mathrm{F}, \mathrm{G}, \mathrm{N}$ & \\
\hline $\begin{array}{l}\text { Pyrethrin, piperonyl } \\
\text { butoxide \& silicon dioxide }\end{array}$ & Diatect II Multipurpose & $\mathrm{F}, \mathrm{G}, \mathrm{N}$ & \\
\hline $\begin{array}{l}\text { Sodium tetraborohydrate } \\
\text { decahydrate }\end{array}$ & Prev-AM & $\mathrm{F}, \mathrm{N}$ & $\begin{array}{l}\text { Do not mix with chemicals containing sulfur or } \\
\text { oils. Do not add adjuvants to Prev-Am. }\end{array}$ \\
\hline Sorbitol octanoate & SorbiShield 90 & $F, G, N$ & $\begin{array}{l}\text { A contact insecticide with limited residual } \\
\text { activity. }\end{array}$ \\
\hline Sucrose octanoate & SucraShield & $\mathrm{F}, \mathrm{G}, \mathrm{N}$ & $\begin{array}{l}\text { A contact insecticide with limited residual } \\
\text { activity. }\end{array}$ \\
\hline Thiamethoxam & Actara & $\mathrm{F}$ & \\
\hline $\begin{array}{l}\text { Thiamethoxam \& } \\
\text { chlorantraniliprole }\end{array}$ & Voliam Flexi & $\mathrm{F}$ & \\
\hline \multicolumn{4}{|c|}{$\begin{array}{l}\text { Footnotes: } \\
1 \text { "F" indicates field production. "G" indicates greenhouse production. “N" indicates nursery production. } \\
{ }^{2} \text { Notes are taken from product labels and restrict use to the condition indicated (suppression, beet armyworm, exposed thrips, etc.), limit } \\
\text { number and patterns of applications, provide phytotoxicity precautions, etc. } \\
{ }^{3} \text { Product is a restricted-use pesticide. } \\
{ }^{4} \text { Product label indicates use in organic production. }\end{array}$} \\
\hline
\end{tabular}


Table 3. Armyworm pesticidal control measures available for commercial strawberry production in Florida. See also caterpillars (Table 5).

\begin{tabular}{|c|c|c|c|}
\hline Common Name & $\begin{array}{l}\text { Trade Name/ } \\
\text { Formulation }\end{array}$ & Production Site $^{1}$ & Notes from labels ${ }^{2}$ \\
\hline Azadirachtin & $\begin{array}{l}\text { Aza-Direct } \\
\text { Azatin XL } \\
\text { Neemix } 4.5^{3}\end{array}$ & $\mathrm{~F}, \mathrm{G}, \mathrm{N}$ & $\begin{array}{l}\text { Will not control adult insects. Effective on all larval stages } \\
\text { and pupae. Reduces damage by repelling and deterring } \\
\text { feeding of all stages of insect. }\end{array}$ \\
\hline Bacillus thuringiensis aizawai & $\begin{array}{l}\text { Agree WG }{ }^{3} \\
\text { XenTari Dry Flowable }\end{array}$ & $\mathrm{F}, \mathrm{G}, \mathrm{N}$ & $\begin{array}{l}\text { For best results apply full coverage sprays when } 1 \text { st or } \\
2 \text { nd instar larvae are present. Where } 4 \text { th or } 5 \text { th instar } \\
\text { larvae are present, a contact insecticide should be used in } \\
\text { combination to enhance control. }\end{array}$ \\
\hline Bacillus thuringiensis kurstaki & $\begin{array}{l}\text { Deliver } \mathrm{LC}^{3} \\
\text { Javelin } \mathrm{WG}^{3} \\
\text { Biobit } \mathrm{HP}^{3} \\
\text { Dipel } \mathrm{DF}^{3} \\
\text { Dipel ES } \\
\text { Lepinox WDG } \\
\text { Crymax Bioinsecticide }\end{array}$ & $\mathrm{F}, \mathrm{G}, \mathrm{N}$ & $\begin{array}{l}\text { May be used to control armyworms or podworms ( } 1 \text { st and } \\
\text { 2nd instar) when populations are light and full coverage } \\
\text { sprays are applied. If mature worms or heavy populations } \\
\text { are present, a contact insecticide should be used to } \\
\text { enhance control. }\end{array}$ \\
\hline $\begin{array}{l}\text { Beauveria bassiana } \\
\text { ATCC } 74040\end{array}$ & Naturalis L & $\mathrm{F}, \mathrm{G}, \mathrm{N}$ & $\begin{array}{l}\text { At least 3-5 applications may be necessary before pests } \\
\text { are adequately under control. Do not tank mix with } \\
\text { fungicides. Wait a minimum of } 48 \text { hours after application } \\
\text { before applying fungicides. }\end{array}$ \\
\hline Bifenthrin & Brigade WSB ${ }^{4}$ & $\mathrm{~F}, \mathrm{~N}$ & $\begin{array}{l}\text { Plants for which bifenthrin tolerances exist may be } \\
\text { rotated at any time. All other crops may be rotated } 30 \\
\text { days after the final application of bifenthrin. }\end{array}$ \\
\hline Bifenthrin \& avermectin B1 & Athena ${ }^{4}$ & $\mathrm{~F}$ & $\begin{array}{l}\text { Not for beet armyworm. Do not make more than two } \\
\text { consecutive applications and four applications per } \\
\text { growing season. Plant back restrictions: For crops that } \\
\text { have bifenthrin and avermectin tolerances, the plant back } \\
\text { is immediate. All other crops may be rotated } 30 \text { days after } \\
\text { the final application. }\end{array}$ \\
\hline Bifenthrin \& imidacloprid & Brigadier $^{4}$ & $\mathrm{~F}, \mathrm{~N}$ & $\begin{array}{l}\text { Not for beet armyworm. Do not apply during or within } \\
10 \text { days after bloom or when bees are actively foraging. } \\
\text { Long, 7-day pre-harvest interval (PHI). Plant back } \\
\text { restrictions: Plants that have tolerances for both bifenthrin } \\
\text { and imidacloprid may be rotated at any time. Crops that } \\
\text { have tolerances for bifenthrin and not imidacloprid can be } \\
\text { rotated } 12 \text { months after the final application of Brigadier } \\
\text { insecticide. Crops that have tolerances for imidacloprid } \\
\text { and not bifenthrin may be rotated } 30 \text { days after the final } \\
\text { application [label has list of crops]. }\end{array}$ \\
\hline Carbaryl & $\begin{array}{l}\text { Cutworm \& Cricket Bait } \\
\text { Sevin Brand 4F } \\
\text { Sevin Brand XLR Plus }\end{array}$ & $\mathrm{F}, \mathrm{N}$ & $\begin{array}{l}\text { Do not apply more than a total of } 10 \text { quarts per acre per } \\
\text { crop. Repeat applications as necessary up to a total of five } \\
\text { times but not more often than once every } 7 \text { days. Plant } \\
\text { back: Do not plant rotational food and feed crops not } \\
\text { listed on this or other carbaryl labels in carbaryl-treated } \\
\text { soil. Long, 7-day pre-harvest interval (PHI). }\end{array}$ \\
\hline Chlorantraniliprole & Coragen & $\mathrm{F}$ & $\begin{array}{l}\text { Beet armyworm; make no more than four applications per } \\
\text { acre per crop. }\end{array}$ \\
\hline Chromobacterium subtsugae & MBI-203 EP ${ }^{3}$ & $\mathrm{~F}, \mathrm{G}, \mathrm{N}$ & \\
\hline Fenpropathrin & Danitol 2.4EC ${ }^{4}$ & $\mathrm{~F}, \mathrm{~N}$ & $\begin{array}{l}\text { Fall \& yellowstriped armyworm; do not exceed more than } \\
\text { two applications totaling } 2-2 / 3 \text { pts. ( } 0.8 \text { lb. a.i.) per acre to } \\
\text { the same planting in } 12 \text { consecutive months. }\end{array}$ \\
\hline Flubendiamide & Synapse WG & $\mathrm{F}, \mathrm{N}$ & \\
\hline
\end{tabular}




\begin{tabular}{|c|c|c|c|}
\hline Common Name & $\begin{array}{l}\text { Trade Name/ } \\
\text { Formulation }\end{array}$ & Production Site ${ }^{1}$ & Notes from labels ${ }^{2}$ \\
\hline Methoxyfenozide & Intrepid 2F & $\mathrm{F}, \mathrm{N}$ & $\begin{array}{l}\text { Do not use more than } 12 \mathrm{fl} \text { oz per acre per application } \\
\text { or } 64 \mathrm{fl} \text { oz of Intrepid } 2 \mathrm{~F}(1 \mathrm{lb} \text { a.i.) per acre per season. } \\
\text { Rotational crop restrictions (plant back): Crops with } \\
\text { registered uses may be replanted at any time. All other } \\
\text { crops grown for food or feed may be replanted after } 7 \\
\text { days. }\end{array}$ \\
\hline Novaluron & Rimon 0.83EC & $\mathrm{F}, \mathrm{N}$ & Does not kill adult insects. \\
\hline Pyrethrin & $\begin{array}{l}\text { PyGanic Crop } \\
\text { Protection EC } 1.4^{3} \\
\text { PyGanic EC } 5.0^{3}\end{array}$ & $\mathrm{~F}, \mathrm{G}, \mathrm{N}$ & \\
\hline $\begin{array}{l}\text { Pyrethrin \& piperonyl } \\
\text { butoxide }\end{array}$ & $\begin{array}{l}\text { EverGreen EC 60-6 } \\
\text { Pyreth-It } \\
\text { Pyrenone Crop Spray }\end{array}$ & $\mathrm{F}, \mathrm{G}, \mathrm{N}$ & \\
\hline $\begin{array}{l}\text { Pyrethrin, piperonyl butoxide } \\
\text { \& silicon dioxide }\end{array}$ & Diatect II Multipurpose & $\mathrm{F}, \mathrm{G}, \mathrm{N}$ & \\
\hline Spinosad & $\begin{array}{l}\text { Entrust }^{3} \\
\text { SpinTor 2SC }\end{array}$ & $\mathrm{F}, \mathrm{N}$ & $\begin{array}{l}\text { Resistance management: Rotate to a different class of } \\
\text { insect control products after two successive applications. } \\
\text { Do not make more than five applications per year. }\end{array}$ \\
\hline Spinetoram & Radiant SC & $\mathrm{F}, \mathrm{N}$ & $\begin{array}{l}\text { Resistance Management: Do not make more than } \\
\text { two consecutive applications of group } 5 \text { insecticides } \\
\text { (spinetoram and spinosad). Do not make more than five } \\
\text { applications per calendar year. }\end{array}$ \\
\hline Steinernema carpocapsae & Millenium & $\mathrm{F}, \mathrm{G}, \mathrm{N}$ & Ground dwelling insects and borers \\
\hline $\begin{array}{l}\text { Thiamethoxam \& } \\
\text { chlorantraniliprole }\end{array}$ & Voliam Flexi & $\mathrm{F}$ & Beet armyworm. \\
\hline \multicolumn{4}{|c|}{ 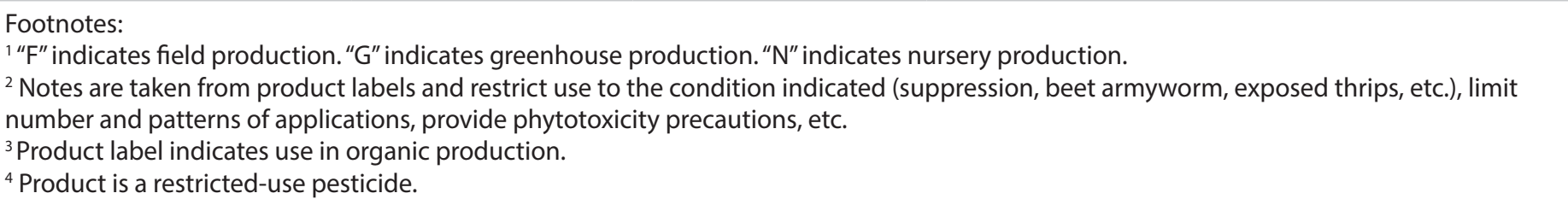 } \\
\hline
\end{tabular}


Table 4. Beetle and weevil pesticidal control measures available for commercial strawberry production in Florida. See also grubs (Table 8).

\begin{tabular}{|c|c|c|c|}
\hline Common Name & $\begin{array}{l}\text { Trade Name/ } \\
\text { Formulation }\end{array}$ & Production Site ${ }^{1}$ & Notes from labels ${ }^{2}$ \\
\hline Acetamiprid & Assail 30SG & $\mathrm{F}, \mathrm{G}, \mathrm{N}$ & $\begin{array}{l}\text { Flea beetle, sap beetles; do not make more than two applications } \\
\text { per growing season. }\end{array}$ \\
\hline Azadirachtin & $\begin{array}{l}\text { Aza-Direct } \\
\text { Azatin } \mathrm{XL} \\
\text { Neemix } 4.5^{3} \\
\text { Ecozin Plus } 1.2 \% \mathrm{ME}^{3}\end{array}$ & $\mathrm{~F}, \mathrm{G}, \mathrm{N}$ & $\begin{array}{l}\text { Will not control adult insects. Effective on all larval stages and } \\
\text { pupae. Reduces damage by repelling and deterring feeding of all } \\
\text { stages of insect. }\end{array}$ \\
\hline Beauveria bassiana & $\begin{array}{l}\text { Naturalis L } \\
\text { BotaniGard ES } \\
\text { Mycotrol O } O^{3}\end{array}$ & $\mathrm{~F}, \mathrm{G}, \mathrm{N}$ & $\begin{array}{l}\text { Typically it takes } 7-10 \text { days after the first spray to see control. } \\
\text { At least } 3-5 \text { applications may be necessary before pests are } \\
\text { adequately under control. See labels for precautions about use with } \\
\text { fungicides. }\end{array}$ \\
\hline Bifenthrin & Brigade WSB ${ }^{4}$ & $\mathrm{~F}, \mathrm{~N}$ & $\begin{array}{l}\text { Flea beetles; strawberry sap beetle. Plants for which bifenthrin } \\
\text { tolerances exist may be rotated at any time. All other crops may be } \\
\text { rotated } 30 \text { days after the final application of bifenthrin. }\end{array}$ \\
\hline Bifenthrin \& avermectin B1 & Athena $^{4}$ & $\mathrm{~F}$ & $\begin{array}{l}\text { Flea beetles, strawberry sap beetles, strawberry root weevil, } \\
\text { strawberry clipper; do not make more than two consecutive } \\
\text { applications and four applications per growing season. Plant } \\
\text { back restrictions: For crops that have bifenthrin and avermectin } \\
\text { tolerances, the plant back is immediate. All other crops may be } \\
\text { rotated } 30 \text { days after the final application. }\end{array}$ \\
\hline Bifenthrin \& imidacloprid & Brigadier $^{4}$ & $\mathrm{~F}, \mathrm{~N}$ & $\begin{array}{l}\text { Flea beetle spp.; do not apply during or within } 10 \text { days after bloom } \\
\text { or when bees are actively foraging. Long, 7-day pre-harvest interval } \\
\text { (PHI). Plant back restrictions: Plants that have tolerances for both } \\
\text { bifenthrin and imidacloprid may be rotated at any time. Crops that } \\
\text { have tolerances for bifenthrin and not imidacloprid can be rotated } \\
12 \text { months after the final application of Brigadier insecticide. Crops } \\
\text { that have tolerances for imidacloprid and not bifenthrin may be } \\
\text { rotated } 30 \text { days after the final application [label has list of crops]. }\end{array}$ \\
\hline Carbaryl & $\begin{array}{l}\text { Carbaryl } 4 \mathrm{~L} \\
\text { Sevin Brand } 4 \mathrm{~F} \\
\text { Sevin Brand } 80 \mathrm{~S} \\
\text { Sevin XLR Plus }\end{array}$ & $\mathrm{F}, \mathrm{N}$ & $\begin{array}{l}\text { Sap beetles }{ }^{5} \text {; flea beetles; June beetles. Plant back: Do not plant } \\
\text { rotational food and feed crops not listed on this or other carbaryl } \\
\text { labels in carbaryl-treated soil. Long, 7-day pre-harvest interval (PHI). }\end{array}$ \\
\hline Chlorantraniliprole & Coragen & $\mathrm{F}$ & $\begin{array}{l}\text { Japanese beetle adult; make no more than four applications per } \\
\text { acre per crop. }\end{array}$ \\
\hline Chlorpyrifos & $\begin{array}{l}\text { Govern } 4 \mathrm{E}^{4} \\
\text { Lorsban Advanced }^{4}\end{array}$ & $\mathrm{~F}, \mathrm{~N}$ & $\begin{array}{l}\text { Strawberry bud weevil; pre-bloom use only. Do not make more than } \\
\text { two applications (foliar) per year. }\end{array}$ \\
\hline Fenpropathrin & Danitol $2.4 \mathrm{EC}^{4}$ & $\mathrm{~F}, \mathrm{~N}$ & $\begin{array}{l}\text { Strawberry sap beetle, strawberry bud weevil (strawberry clipper); } \\
\text { do not exceed more than two applications totaling } 2-2 / 3 \text { pts. }(0.8 \\
\text { lbs. a.i.) per acre to the same planting in } 12 \text { consecutive months. }\end{array}$ \\
\hline Imidacloprid & $\begin{array}{l}\text { Admire Pro } \\
\text { Couraze } 2 \mathrm{~F}\end{array}$ & $\mathrm{~F}, \mathrm{~N}$ & $\begin{array}{l}\text { Post-harvest use on perennial strawberry: White grub complex } \\
\text { (grubs of Asiatic garden beetle, European chafer, masked chafer, } \\
\text { Japanese beetle, Oriental beetle). Long, 14-day pre-harvest interval } \\
\text { (PHI). }\end{array}$ \\
\hline $\begin{array}{l}\text { Isaria fumosorosea } \\
\text { (formerly Paecilomyces } \\
\text { fumosoroseus) }\end{array}$ & $\begin{array}{l}\text { Preferal } \\
\text { PFR-97 20\% WDG }{ }^{3}\end{array}$ & $\mathrm{~F}, \mathrm{G}, \mathrm{N}$ & $\begin{array}{l}\text { Coleoptera grubs and larvae; most effective when relative humidity } \\
\text { is } 80 \% \text { or higher for } 8-10 \text { hours. Can be mixed with copper-based } \\
\text { fungicides; do not mix with other fungicides or apply within } 5 \text { days } \\
\text { of fungicide applications other than copper. }\end{array}$ \\
\hline Malathion & $\begin{array}{l}\text { Gowan Malathion } 8 \mathrm{~F} \\
\text { Malathion 5EC }\end{array}$ & $\mathrm{F}, \mathrm{N}$ & Strawberry root weevil. \\
\hline Novaluron & Rimon $0.83 \mathrm{EC}$ & $\mathrm{F}, \mathrm{N}$ & Sap beetle larvae; does not kill adult insects. \\
\hline Pyrethrin & $\begin{array}{l}\text { PyGanic Crop } \\
\text { Protection EC } 1.4^{3} \\
\text { PyGanic EC } 5.0^{3}\end{array}$ & $\mathrm{~F}, \mathrm{G}, \mathrm{N}$ & \\
\hline
\end{tabular}




\begin{tabular}{|c|c|c|c|}
\hline Common Name & $\begin{array}{l}\text { Trade Name/ } \\
\text { Formulation }\end{array}$ & Production Site ${ }^{1}$ & Notes from labels ${ }^{2}$ \\
\hline $\begin{array}{l}\text { Pyrethrin \& } \\
\text { Piperonyl Butoxide }\end{array}$ & $\begin{array}{l}\text { Evergreen EC 60-6 } \\
\text { Pyreth-It } \\
\text { Pyrenone Crop Spray }\end{array}$ & $\mathrm{F}, \mathrm{G}, \mathrm{N}$ & Cucumber beetles, flea beetles, corn sap beetle. \\
\hline $\begin{array}{l}\text { Pyrethrin, rotenone \& } \\
\text { associated resins }\end{array}$ & Pyrellin E.C. & $\mathrm{F}, \mathrm{G}, \mathrm{N}$ & Strawberry root worms. \\
\hline Steinernema carpocapsae & Millenium & $\mathrm{F}, \mathrm{G}, \mathrm{N}$ & $\begin{array}{l}\text { Ground-dwelling insects and borers: Blackvine weevil, strawberry } \\
\text { root weevil. }\end{array}$ \\
\hline Thiamethoxam & Actara & $\mathrm{F}$ & Weevil (adult). \\
\hline $\begin{array}{l}\text { Thiamethoxam \& } \\
\text { chlorantraniliprole }\end{array}$ & Voliam Flexi & $\mathrm{F}$ & Japanese beetle (adult), weevil (adult). \\
\hline \multicolumn{4}{|c|}{ 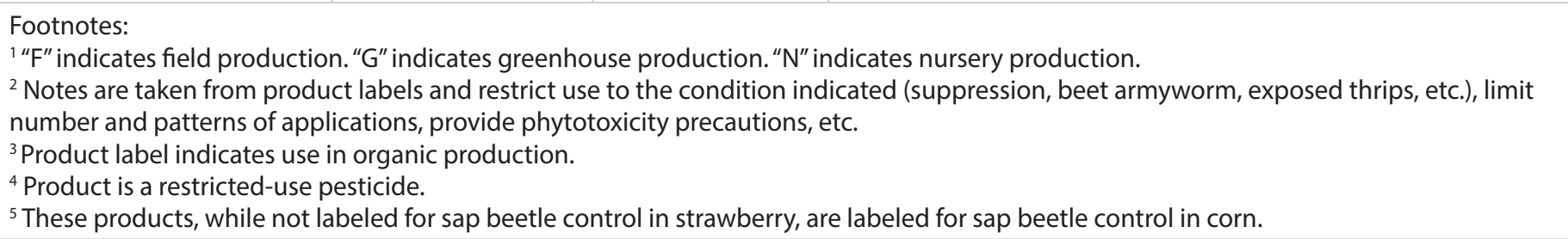 } \\
\hline
\end{tabular}


Table 5. Caterpillar pesticidal control measures available for commercial strawberry production in Florida. (Includes budworms, earworms, leafrollers, leaftiers, lesser cornstalk borer and looper.) Also see armyworms (Table 3).

\begin{tabular}{|c|c|c|c|}
\hline Common Name & $\begin{array}{l}\text { Trade Name/ } \\
\text { Formulation }\end{array}$ & $\begin{array}{l}\text { Production } \\
\text { Site }^{1}\end{array}$ & Notes from labels ${ }^{2}$ \\
\hline Acetamiprid & Assail 30SG & $\mathrm{F}, \mathrm{G}, \mathrm{N}$ & $\begin{array}{l}\text { Obliquebanded leafroller; do not make more than two applications per growing } \\
\text { season. }\end{array}$ \\
\hline Azadirachtin & $\begin{array}{l}\text { Aza-Direct }{ }^{3} \\
\text { Azatin XL } \\
\text { Ecozin Plus } \\
1.2 \% \mathrm{ME}^{3} \\
\text { Neemix } 4.5^{3}\end{array}$ & $\mathrm{~F}, \mathrm{G}, \mathrm{N}$ & $\begin{array}{l}\text { Will not control adult insects. Effective on all larval stages and pupae. Reduces } \\
\text { damage by repelling and deterring feeding of all stages of insect. }\end{array}$ \\
\hline $\begin{array}{l}\text { Bacillus thuringiensis } \\
\text { aizawai strain ABTS- } \\
1857\end{array}$ & $\begin{array}{l}\text { XenTari Dry } \\
\text { Flowable }\end{array}$ & $\mathrm{F}, \mathrm{G}, \mathrm{N}$ & $\begin{array}{l}\text { Loopers, obliquebanded leafroller, omnivorous leafroller, saltmarsh caterpillar, } \\
\text { tobacco budworm. }\end{array}$ \\
\hline $\begin{array}{l}\text { Bacillus thuringiensis } \\
\text { kurstaki }\end{array}$ & $\begin{array}{l}\text { Javelin } \mathrm{WG}^{3} \\
\text { Deliver } \mathrm{LC}^{3} \\
\text { Biobit } \mathrm{HP}^{3} \\
\text { Dipel DF} \\
\text { Dipel ES } \\
\text { Dipel Pro DF }\end{array}$ & $\mathrm{F}, \mathrm{G}, \mathrm{N}$ & $\begin{array}{l}\text { Bollworm, Helicoverpa spp., Heliothis spp., tobacco budworm, tomato fruitworm, } \\
\text { looper, black cutworm, obliquebanded leafroller, omnivorous leafroller, } \\
\text { omnivorous leaftier, saltmarsh caterpillar. }\end{array}$ \\
\hline Beauveria bassiana & $\begin{array}{l}\text { Naturalis L } \\
\text { Mycotrol } \mathrm{O}^{3}\end{array}$ & $\mathrm{~F}, \mathrm{G}, \mathrm{N}$ & $\begin{array}{l}\text { Eggs of lepidopteran pests, leafrollers, lesser cornstalk borer, loopers, tomato } \\
\text { fruitworm; at least 3-5 applications may be necessary before pests are } \\
\text { adequately under control. See labels for precautions about use with fungicides. }\end{array}$ \\
\hline Bifenthrin & Brigade WSB ${ }^{4}$ & $\mathrm{~F}, \mathrm{~N}$ & $\begin{array}{l}\text { Heliothis spp., leafrollers; Plant back restrictions: Plants for which bifenthrin } \\
\text { tolerances exist may be rotated at any time. All other crops may be rotated } 30 \\
\text { days after the final application of bifenthrin. }\end{array}$ \\
\hline $\begin{array}{l}\text { Bifenthrin \& } \\
\text { avermectin B1 }\end{array}$ & Athena ${ }^{4}$ & $\mathrm{~F}$ & $\begin{array}{l}\text { Corn earworm, leafroller, looper, orange tortrix; do not make more than two } \\
\text { consecutive applications and four applications per growing season. Plant back } \\
\text { restrictions: For crops that have bifenthrin and avermectin tolerances, the } \\
\text { plant back is immediate. All other crops may be rotated } 30 \text { days after the final } \\
\text { application. }\end{array}$ \\
\hline $\begin{array}{l}\text { Bifenthrin \& } \\
\text { imidacloprid }\end{array}$ & Brigadier $^{4}$ & $\mathrm{~F}, \mathrm{~N}$ & $\begin{array}{l}\text { Corn earworm; do not apply during or within } 10 \text { days after bloom or when } \\
\text { bees are actively foraging. Long, 7-day pre-harvest interval (PHI). Plant back } \\
\text { restrictions: Plants that have tolerances for both bifenthrin and imidacloprid } \\
\text { may be rotated at any time. Crops that have tolerances for bifenthrin and not } \\
\text { imidacloprid can be rotated } 12 \text { months after the final application of Brigadier } \\
\text { insecticide. Crops that have tolerances for imidacloprid and not bifenthrin may } \\
\text { be rotated } 30 \text { days after the final application [label has list of crops]. }\end{array}$ \\
\hline Carbaryl & $\begin{array}{l}\text { Carbaryl 4L } \\
\text { Sevin Brand 4F } \\
\text { Sevin Brand } \\
\text { XLR Plus }\end{array}$ & $\mathrm{F}, \mathrm{N}$ & $\begin{array}{l}\text { Omnivorous leafroller, omnivorous leaftier, strawberry fruitworm, strawberry } \\
\text { leafroller, saltmarsh caterpillar; do not plant rotational food crops not listed on } \\
\text { this or other carbaryl labels in carbaryl-treated soil. Long, 7-day pre-harvest } \\
\text { interval (PHI). }\end{array}$ \\
\hline Chlorantraniliprole & Coragen & $\mathrm{F}$ & $\begin{array}{l}\text { Cabbage looper, corn earworm; make no more than four applications per acre } \\
\text { per crop. }\end{array}$ \\
\hline Diazinon & $\begin{array}{l}\text { Diazinon AG } \\
500^{4} \\
\text { Diazinon } 50 W^{4} \\
\text { Diazinon AG600 } \\
\text { WBC }^{4}\end{array}$ & $\mathrm{~F}, \mathrm{~N}$ & $\begin{array}{l}\text { Strawberry leafroller; make a maximum of one foliar application per crop and a } \\
\text { maximum of one soil application per crop. Long, 5-day pre-harvest interval (PHI). }\end{array}$ \\
\hline $\begin{array}{l}\text { Edible fish oil \& sesame } \\
\text { oil }\end{array}$ & $\begin{array}{l}\text { Organocide } \\
\text { 3-in-1 Garden } \\
\text { Spray }^{3}\end{array}$ & $\mathrm{~F}, \mathrm{G}, \mathrm{N}$ & Leafrollers. \\
\hline Flubendiamide & Synapse WG & $\mathrm{F}, \mathrm{N}$ & $\begin{array}{l}\text { Corn earworm, cutworm, lesser cornstalk borer, omnivorous leaftier, strawberry } \\
\text { leafroller. }\end{array}$ \\
\hline $\begin{array}{l}\text { Isaria fumosorosea } \\
\text { (formerly Paecilomyces } \\
\text { fumosoroseus) }\end{array}$ & $\begin{array}{l}\text { Preferal } \\
\text { PFR-97 20\% } \\
\text { WDG }^{3}\end{array}$ & $\mathrm{~F}, \mathrm{G}, \mathrm{N}$ & $\begin{array}{l}\text { Lepidoptera caterpillars and larvae; most effective when relative humidity is } 80 \% \\
\text { or higher for } 8-10 \text { hours. Can be mixed with copper-based fungicides; do not mix } \\
\text { with other fungicides or apply within } 5 \text { days of fungicide applications other than } \\
\text { copper. }\end{array}$ \\
\hline
\end{tabular}




\begin{tabular}{|c|c|c|c|}
\hline Common Name & $\begin{array}{l}\text { Trade Name/ } \\
\text { Formulation }\end{array}$ & $\begin{array}{l}\text { Production } \\
\text { Site }^{1}\end{array}$ & Notes from labels ${ }^{2}$ \\
\hline Malathion & $\begin{array}{l}\text { Gowan } \\
\text { Malathion 8F } \\
\text { Malathion 5EC }\end{array}$ & $\mathrm{F}, \mathrm{N}$ & Strawberry leafrollers. \\
\hline Methoxyfenozide & Intrepid 2F & $\mathrm{F}, \mathrm{N}$ & Armyworms; corn earworm suppression. \\
\hline Naled & $\begin{array}{l}\text { Dibrom } 8 \\
\text { Emulsive }^{4}\end{array}$ & $\mathrm{~F}, \mathrm{~N}$ & Leafrollers, omnivorous leaftiers; do not apply when temperature is over $90^{\circ} \mathrm{F}$. \\
\hline Novaluron & Rimon 0.83EC & $\mathrm{F}, \mathrm{N}$ & Corn earworm, loopers, webworms; does not kill adult insects. \\
\hline $\begin{array}{l}\text { Oil (mineral, paraffinic, } \\
\text { petroleum, vegetable, } \\
\text { etc.) }\end{array}$ & Ultra-Fine Oil & $\mathrm{F}, \mathrm{N}$ & Eggs of certain caterpillars; see labels for phytotoxicity precautions. \\
\hline $\begin{array}{l}\text { Polyhedral occlusion } \\
\text { bodies of the nuclear } \\
\text { polyhedrosis virus of } \\
\text { Helicoverpa zea }\end{array}$ & Gemstar LC ${ }^{3}$ & $\mathrm{~F}, \mathrm{~N}$ & $\begin{array}{l}\text { Corn earworm, Helicoverpa zea, cotton bollworm, tomato fruitworm, tobacco } \\
\text { budworm, Heliothis virescens. }\end{array}$ \\
\hline Pyrethrin & $\begin{array}{l}\text { PyGanic Crop } \\
\text { Protection EC- } \\
1.4^{3} \\
\text { PyGanic EC } 5.0^{3}\end{array}$ & $\mathrm{~F}, \mathrm{G}, \mathrm{N}$ & Budworms, loopers. \\
\hline $\begin{array}{l}\text { Pyrethrin \& piperonyl } \\
\text { butoxide }\end{array}$ & $\begin{array}{l}\text { EverGreen EC } \\
60-6 \\
\text { Pyreth-It } \\
\text { Pyrenone Crop } \\
\text { Spray }\end{array}$ & $\mathrm{F}, \mathrm{G}, \mathrm{N}$ & Budworms, loopers, leafrollers, leaftiers, lesser cornstalk borer. \\
\hline $\begin{array}{l}\text { Pyrethrin, piperonyl } \\
\text { butoxide \& silicon } \\
\text { dioxide }\end{array}$ & $\begin{array}{l}\text { Diatect II } \\
\text { Multipurpose }\end{array}$ & $\mathrm{F}, \mathrm{G}, \mathrm{N}$ & Cabbage looper, strawberry leafrollers. \\
\hline $\begin{array}{l}\text { Pyrethrin, rotenone \& } \\
\text { associated resins }\end{array}$ & Pyrellin E.C. & $\mathrm{F}, \mathrm{G}, \mathrm{N}$ & Strawberry leafrollers. \\
\hline Sorbitol octanoate & SorbiShield 90 & $\mathrm{~F}, \mathrm{G}, \mathrm{N}$ & A contact insecticide with limited residual activity. \\
\hline Spinosad & $\begin{array}{l}\text { Entrust }^{3} \\
\text { SpinTor 2SC }\end{array}$ & $\mathrm{F}, \mathrm{G}, \mathrm{N}$ & Leafrollers. \\
\hline $\begin{array}{l}\text { Steinernema } \\
\text { carpocapsae }\end{array}$ & Millenium & $\mathrm{F}, \mathrm{G}, \mathrm{N}$ & Ground-dwelling insects and borers; Armyworms. \\
\hline $\begin{array}{l}\text { Thiamethoxam \& } \\
\text { chlorantraniliprole }\end{array}$ & Voliam Flexi & $\mathrm{F}$ & Cabbage looper, corn earworm. \\
\hline \multicolumn{4}{|c|}{ 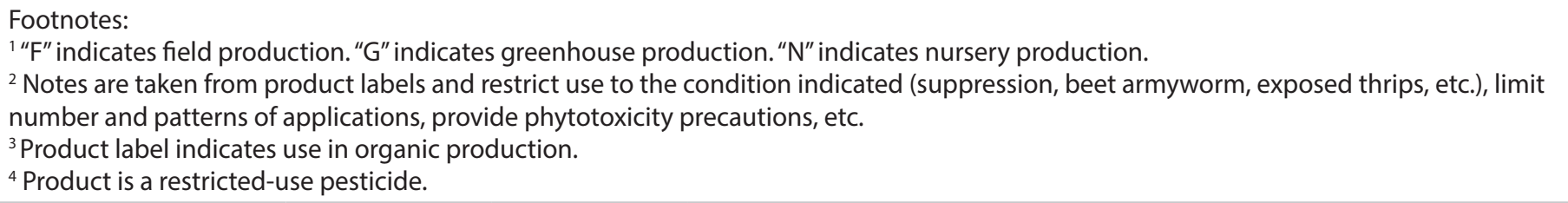 } \\
\hline
\end{tabular}


Table 6. Cricket pesticidal control measures available for commercial strawberry production in Florida. See also mole cricket (Table 10).

\begin{tabular}{|l|l|l|l|}
\hline Common Name & Trade Name/ Formulation & Production Site & Notes from labels \\
\hline Carbaryl & Cutworm \& Cricket Bait & F, N & $\begin{array}{l}\text { Field crickets. Long, 7-day pre-harvest interval } \\
\text { (PHI). }\end{array}$ \\
\hline Malathion & $\begin{array}{l}\text { Gowan Malathion 8F } \\
\text { Malathion 5EC }\end{array}$ & Field crickets. \\
\hline Pyrethrin & $\begin{array}{l}\text { PyGanic Crop Protection EC } \\
1.4^{3} \\
\text { PyGanic EC 5.03 }\end{array}$ & F, G, N & \\
\hline Pyrethrin \& piperonyl butoxide & $\begin{array}{l}\text { EverGreen EC 60-6 } \\
\text { Pyreth-It } \\
\text { Pyrenone Crop Spray }\end{array}$ & F, G, N & \\
\hline $\begin{array}{l}\text { Footnotes: } \\
\text { 1 "F" indicates field production. “G" indicates greenhouse production. “N” indicates nursery production. } \\
\text { 2 Notes are taken from product labels and restrict use to the condition indicated (suppression, beet armyworm, exposed thrips, etc.), limit } \\
\text { number and patterns of applications, provide phytotoxicity precautions, etc. } \\
\text { 3Product label indicates use in organic production. }\end{array}$ & \\
\hline
\end{tabular}


Table 7. Fruit fly (vinegar fly) and spotted wing drosophila pesticidal control measures available for commercial strawberry production in Florida.

\begin{tabular}{|c|c|c|c|}
\hline Common Name & $\begin{array}{l}\text { Trade Name/ } \\
\text { Formulation }\end{array}$ & Production Site ${ }^{1}$ & Notes from labels ${ }^{2}$ \\
\hline Azadirachtin & $\begin{array}{l}\text { Aza-Direct } \\
\text { Azatin XL } \\
\text { Ecozin Plus } 1.2 \% \mathrm{ME}^{3} \\
\text { Neemix } 4.5^{3}\end{array}$ & $\mathrm{~F}, \mathrm{G}, \mathrm{N}$ & $\begin{array}{l}\text { Will not control adult insects. Effective on all larval stages and } \\
\text { pupae. Reduces damage by repelling and deterring feeding of all } \\
\text { stages of insect. }\end{array}$ \\
\hline Bifenthrin ${ }^{4}$ & Brigade WSB ${ }^{5}$ & $\mathrm{~F}, \mathrm{~N}$ & $\begin{array}{l}\text { Plant back restrictions: Plants for which bifenthrin tolerances exist } \\
\text { may be rotated at any time. All other crops may be rotated } 30 \\
\text { days after the final application of bifenthrin. }\end{array}$ \\
\hline Fenpropathrin ${ }^{4}$ & Danitol 2.4EC & $\mathrm{F}, \mathrm{N}$ & $\begin{array}{l}\text { Do not exceed more than two applications to the same planting } \\
\text { in } 12 \text { consecutive months. }\end{array}$ \\
\hline Malathion ${ }^{4}$ & $\begin{array}{l}\text { Gowan Malathion } 8 \mathrm{~F} \\
\text { Malathion } 5 \mathrm{EC}\end{array}$ & $\mathrm{F}, \mathrm{N}$ & \\
\hline Naled $^{4}$ & Dibrom 8 Emulsive ${ }^{5}$ & $\mathrm{~F}, \mathrm{~N}$ & Do not apply when temperature is over $90^{\circ} \mathrm{F}$. \\
\hline Pyrethrin & $\begin{array}{l}\text { PyGanic Crop Protection } \\
\text { EC } 1.4^{3} \\
\text { PyGanic EC } 5.0^{3}\end{array}$ & $\mathrm{~F}, \mathrm{G}, \mathrm{N}$ & Vinegar flies. \\
\hline $\begin{array}{l}\text { Pyrethrin \& piperonyl } \\
\text { butoxide }\end{array}$ & $\begin{array}{l}\text { Pyreth-It } \\
\text { EverGreen EC 60-6 } \\
\text { Pyrenone Crop Spray }\end{array}$ & $\mathrm{F}, \mathrm{G}, \mathrm{N}$ & $\begin{array}{l}\text { Fruit flies; vinegar flies; for use on harvested fruits and vegetables: } \\
\text { To control Drosophila spp. fruit flies. }\end{array}$ \\
\hline $\begin{array}{l}\text { Pyrethrin, piperonyl } \\
\text { butoxide \& silicon } \\
\text { dioxide }\end{array}$ & Diatect II Multipurpose & $\mathrm{F}, \mathrm{G}, \mathrm{N}$ & Fruit flies. \\
\hline Spinetoram ${ }^{4}$ & Radiant SC & $\mathrm{F}, \mathrm{N}$ & $\begin{array}{l}\text { Resistance Management: Do not make more than two } \\
\text { consecutive applications of group } 5 \text { insecticides (spinetoram and } \\
\text { spinosad). Do not make more than five applications per calendar } \\
\text { year. }\end{array}$ \\
\hline Spinosad $^{4}$ & $\begin{array}{l}\text { GF-120 NF Naturalyte } \\
\text { Fruit Fly Bait }{ }^{3}\end{array}$ & $\mathrm{~F}, \mathrm{~N}$ & Tephritid fruit flies. \\
\hline \multicolumn{4}{|c|}{$\begin{array}{l}\text { Footnotes: } \\
1 \text { "F" indicates field production. "G" indicates greenhouse production. "N" indicates nursery production. } \\
{ }^{2} \text { Notes are taken from product labels and restrict use to the condition indicated (suppression, beet armyworm, exposed thrips, etc.), limit } \\
\text { number and patterns of applications, provide phytotoxicity precautions, etc. } \\
{ }^{3} \text { Product label indicates use in organic production. } \\
{ }^{4} \text { These products do not make a label claim for drosophila control but have been shown to be useful to manage spotted wing drosophila. } \\
{ }^{5} \text { Product is a restricted-use pesticide. }\end{array}$} \\
\hline
\end{tabular}


Table 8. Grub pesticidal control measures available for commercial strawberry production in Florida. See also beetles (Table 4).

\begin{tabular}{|c|c|c|c|}
\hline Common Name & Trade Name/ Formulation & Production Site ${ }^{1}$ & Notes from labels ${ }^{2}$ \\
\hline Azadirachtin & $\begin{array}{l}\text { Azatin XL } \\
\text { Neemix } 4.5^{3}\end{array}$ & $\mathrm{~F}, \mathrm{G}, \mathrm{N}$ & $\begin{array}{l}\text { Effective on all larval stages and pupae. } \\
\text { Reduces damage by repelling and } \\
\text { deterring feeding of all stages of insect. }\end{array}$ \\
\hline Beauveria bassiana strain GHA & $\begin{array}{l}\text { BotaniGard ES } \\
\text { Mycotrol O }\end{array}$ & $\mathrm{F}, \mathrm{G}, \mathrm{N}$ & $\begin{array}{l}\text { Typically it takes } 7-10 \text { days after the } \\
\text { first spray to see control. See labels for } \\
\text { precautions about use with fungicides. }\end{array}$ \\
\hline Chlorpyrifos & $\begin{array}{l}\text { Govern 4E } \\
\text { Lorsban } 75 W G\end{array}$ & $\mathrm{~F}, \mathrm{~N}$ & $\begin{array}{l}\text { Grub; do not make more than one (pre- } \\
\text { plant) application per year. }\end{array}$ \\
\hline Heterorhabditis bacteriophora & Nemasys G & $\mathrm{F}, \mathrm{G}, \mathrm{N}$ & $\begin{array}{l}\text { European chafer, oriental beetle \& } \\
\text { Japanese beetle grubs. }\end{array}$ \\
\hline Methyl bromide \& chloropicrin & MBC $98-2^{4}$ & $\mathrm{~F}, \mathrm{G}, \mathrm{N}$ & Pre-plant. \\
\hline Steinernema carpocapsae & Millenium & $\mathrm{F}, \mathrm{G}, \mathrm{N}$ & $\begin{array}{l}\text { Ground-dwelling insects and borers: } \\
\text { Strawberry root weevil. }\end{array}$ \\
\hline Thiamethoxam & Platinum & $\mathrm{F}$ & Pre-harvest interval is 50 days. \\
\hline \multicolumn{4}{|c|}{$\begin{array}{l}\text { Footnotes: } \\
1 \text { "F" indicates field production. "G" indicates greenhouse production. "N" indicates nursery production. } \\
{ }^{2} \text { Notes are taken from product labels and restrict use to the condition indicated (suppression, beet armyworm, exposed thrips, etc.), limit } \\
\text { number and patterns of applications, provide phytotoxicity precautions, etc. } \\
{ }^{3} \text { Product label indicates use in organic production. } \\
{ }^{4} \text { Product is a restricted-use pesticide. }\end{array}$} \\
\hline
\end{tabular}


Table 9. Mite pesticidal control measures available for commercial strawberry production in Florida. (Includes spider mites, cyclamen mites, and rust mites.)

\begin{tabular}{|c|c|c|c|}
\hline Common Name & $\begin{array}{l}\text { Trade Name/ } \\
\text { Formulation }\end{array}$ & Production Site $^{1}$ & Notes from labels ${ }^{2}$ \\
\hline Abamectin & $\begin{array}{l}\text { Agri-Mek } 0.15 \mathrm{EC}^{3} \\
\text { Temprano }\end{array}$ & $\mathrm{F}$ & $\begin{array}{l}\text { Twospotted spider mite, strawberry spider mite, suppression of cyclamen } \\
\text { mite. }\end{array}$ \\
\hline Acequinocyl & Kanemite 15 SC & $\mathrm{F}, \mathrm{N}$ & Twospotted spider mite (Tetranychus urticae). \\
\hline Azadirachtin & $\begin{array}{l}\text { Aza-Direct }{ }^{4} \\
\text { Azahar }\end{array}$ & $F, G, N$ & Will not control adults. \\
\hline $\begin{array}{l}\text { Beauveria bassiana } \\
\text { ATCC } 74040\end{array}$ & Naturalis L & $\mathrm{F}, \mathrm{G}, \mathrm{N}$ & $\begin{array}{l}\text { At least 3-5 applications may be necessary before pests are adequately } \\
\text { under control. Do not tank mix with fungicides. Wait a minimum of } 48 \\
\text { hours after application before applying fungicides. }\end{array}$ \\
\hline Bifenazate & Acramite 50WS & $\mathrm{F}, \mathrm{N}$ & $\begin{array}{l}\text { Twospotted spider mite; strawberry mite; two sprays is the total number } \\
\text { of sprays per season. Nursery use must be on plants that will not bear fruit } \\
\text { within } 1 \text { year of application. }\end{array}$ \\
\hline Bifenthrin & Brigade WSB ${ }^{3}$ & $\mathrm{~F}, \mathrm{~N}$ & $\begin{array}{l}\text { Spider mites; plants for which bifenthrin tolerances exist may be } \\
\text { rotated at any time. All other crops may be rotated } 30 \text { days after the } \\
\text { final application of bifenthrin. Plant back restrictions: Plants for which } \\
\text { bifenthrin tolerances exist may be rotated at any time. All other crops may } \\
\text { be rotated } 30 \text { days after the final application of bifenthrin. }\end{array}$ \\
\hline $\begin{array}{l}\text { Bifenthrin \& } \\
\text { avermectin B1 }\end{array}$ & Athena ${ }^{3}$ & $\mathrm{~F}$ & $\begin{array}{l}\text { Cyclamen mite, strawberry mite, twospotted spider mite; do not make } \\
\text { more than two consecutive applications and four applications per } \\
\text { growing season. Plant back restrictions: For crops that have bifenthrin and } \\
\text { avermectin tolerances, the plant back is immediate. All other crops may } \\
\text { be rotated } 30 \text { days after the final application. }\end{array}$ \\
\hline $\begin{array}{l}\text { Chromobacterium } \\
\text { subtsugae }\end{array}$ & $\mathrm{MBI}-203 \mathrm{EP}^{4}$ & $\mathrm{~F}, \mathrm{G}, \mathrm{N}$ & \\
\hline Diazinon & $\begin{array}{l}\text { Diazinon AG-50033 } \\
\text { Diazinon AG600 } \\
\text { WBC }^{3} \\
\text { Diazinon } 50 W^{3}\end{array}$ & $\mathrm{~F}, \mathrm{~N}$ & $\begin{array}{l}\text { For cyclamen mites, direct spray to plant crowns. Make a maximum of } \\
\text { one foliar application per crop and a maximum of one soil application per } \\
\text { crop. Long, 5-day pre-harvest interval (PHI). }\end{array}$ \\
\hline $\begin{array}{l}\text { Edible fish oil \& } \\
\text { sesame oil }\end{array}$ & $\begin{array}{l}\text { Organocide } 3 \text {-in-1 } \\
\text { Garden Spray }\end{array}$ & $\mathrm{F}, \mathrm{G}, \mathrm{N}$ & Spider mites. \\
\hline Etoxazole & Zeal Miticide & $\mathrm{F}, \mathrm{N}$ & $\begin{array}{l}\text { Twospotted spider mite; do not make more than one Zeal application per } \\
\text { growing season. }\end{array}$ \\
\hline Fenbutatin-oxide & Vendex 50WP ${ }^{3}$ & $\mathrm{~F}, \mathrm{~N}$ & Twospotted spider mite; make no more than two applications per season. \\
\hline Fenpropathrin & Danitol 2.4EC ${ }^{3}$ & $\mathrm{~F}, \mathrm{~N}$ & $\begin{array}{l}\text { Twospotted spider mite; do not exceed more than two applications to the } \\
\text { same planting in } 12 \text { consecutive months. }\end{array}$ \\
\hline Fenpyroximate & Portal & $\mathrm{F}, \mathrm{N}$ & $\begin{array}{l}\text { Broad mite, citrus rust mite, cyclamen mite, twospotted spider mite; do } \\
\text { not make more than two applications per season. }\end{array}$ \\
\hline Hexythiazox & Savey 50 DF & $\mathrm{F}$ & Twospotted spider mite; do not make more than one application per year. \\
\hline $\begin{array}{l}\text { Isaria fumosorosea } \\
\text { (formerly } \\
\text { Paecilomyces } \\
\text { fumosoroseus) }\end{array}$ & $\begin{array}{l}\text { Preferal } \\
\text { PFR-97 20\% WDG }{ }^{4}\end{array}$ & $\mathrm{~F}, \mathrm{G}, \mathrm{N}$ & $\begin{array}{l}\text { Spider mites, broad mites, rust mites; most effective when relative } \\
\text { humidity is } 80 \% \text { or higher for } 8-10 \text { hours. Can be mixed with copper- } \\
\text { based fungicides; do not mix with other fungicides or apply within } 5 \text { days } \\
\text { of fungicide applications other than copper. }\end{array}$ \\
\hline Naled & Dibrom 8 Emulsive 3 & $\mathrm{~F}, \mathrm{~N}$ & Spider mites; do not apply when temperature is over $90^{\circ} \mathrm{F}$. \\
\hline Neem oil & Trilogy $^{4}$ & $\mathrm{~F}, \mathrm{~N}$ & $\begin{array}{l}\text { Spider mites; avoid tank mixes with captan, sulfur, or other chemically } \\
\text { similar products because unpredictable results or leaf burn may occur. }\end{array}$ \\
\hline $\begin{array}{l}\text { Oil (mineral, } \\
\text { paraffinic, } \\
\text { petroleum, } \\
\text { vegetable, etc.) }\end{array}$ & $\begin{array}{l}\text { Mite-E-Oil } \\
\text { Ultra-Pure Oil }\end{array}$ & $\mathrm{F}, \mathrm{N}$ & See labels for phytotoxicity precautions. \\
\hline $\begin{array}{l}\text { Potassium salts of } \\
\text { fatty acids } \\
\text { (insecticidal soap) }\end{array}$ & $\begin{array}{l}\text { AllPro Insecticidal } \\
\text { Soap } 40 \% \\
\text { M-Pede }\end{array}$ & $\mathrm{F}, \mathrm{G}, \mathrm{N}$ & $\begin{array}{l}\text { Twospotted mites; do not mix with sulfur. See labels for phytotoxicity } \\
\text { precautions. }\end{array}$ \\
\hline Potassium silicate & Sil-MATRIX & $\mathrm{F}, \mathrm{G}, \mathrm{N}$ & Spider mites suppression. \\
\hline
\end{tabular}




\begin{tabular}{|c|c|c|c|}
\hline Common Name & $\begin{array}{l}\text { Trade Name/ } \\
\text { Formulation }\end{array}$ & Production Site ${ }^{1}$ & Notes from labels ${ }^{2}$ \\
\hline Pyrethrin & $\begin{array}{l}\text { PyGanic Crop } \\
\text { Protection EC } 1.4^{4} \\
\text { PyGanic EC 5.0 }\end{array}$ & $F, G, N$ & \\
\hline $\begin{array}{l}\text { Pyrethrin \& } \\
\text { piperonyl butoxide }\end{array}$ & $\begin{array}{l}\text { EverGreen EC 60-6 } \\
\text { Pyrenone Crop Spray }\end{array}$ & $\mathrm{F}, \mathrm{G}, \mathrm{N}$ & Strawberry mites. \\
\hline $\begin{array}{l}\text { Pyrethrin, rotenone } \\
\text { \& associated resins }\end{array}$ & Pyrellin EC & $\mathrm{F}, \mathrm{G}, \mathrm{N}$ & \\
\hline $\begin{array}{l}\text { Pyrethrin, piperonyl } \\
\text { butoxide \& silicon } \\
\text { dioxide }\end{array}$ & $\begin{array}{l}\text { Diatect II } \\
\text { Multipurpose }\end{array}$ & $\mathrm{F}, \mathrm{G}, \mathrm{N}$ & \\
\hline $\begin{array}{l}\text { Sodium } \\
\text { tetraborohydrate } \\
\text { decahydrate }\end{array}$ & Prev-Am & $\mathrm{F}, \mathrm{N}$ & $\begin{array}{l}\text { Do not mix with chemicals containing sulfur or oils. Do not add adjuvants } \\
\text { to Prev-Am. }\end{array}$ \\
\hline Sorbitol octanoate & SorbiShield 90 & $\mathrm{~F}, \mathrm{G}, \mathrm{N}$ & A contact insecticide with limited residual activity. \\
\hline Spiromesifen & Oberon $2 \mathrm{SC}$ & $\mathrm{F}, \mathrm{N}$ & $\begin{array}{l}\text { Twospotted spider mite; maximum number of applications per crop } \\
\text { season is three. }\end{array}$ \\
\hline Sucrose octanoate & SucraShield & $\mathrm{F}, \mathrm{G}, \mathrm{N}$ & A contact insecticide with limited residual activity. \\
\hline \multicolumn{4}{|c|}{$\begin{array}{l}\text { Footnotes: } \\
1 \text { "F" indicates field production. “G" indicates greenhouse production. “ } \mathrm{N} \text { ” indicates nursery production. } \\
{ }^{2} \text { Notes are taken from product labels and restrict use to the condition indicated (suppression, beet armyworm, exposed thrips, etc.), limit } \\
\text { number and patterns of applications, provide phytotoxicity precautions, etc. } \\
{ }^{3} \text { Product is a restricted-use pesticide. } \\
{ }^{4} \text { Product label indicates use in organic production. }\end{array}$} \\
\hline
\end{tabular}

Table 10. Mole cricket pesticidal control measures available for commercial strawberry production in Florida.

\begin{tabular}{|c|c|c|c|}
\hline Common Name & $\begin{array}{l}\text { Trade Name/ } \\
\text { Formulation }\end{array}$ & Production Site ${ }^{1}$ & Notes from labels ${ }^{2}$ \\
\hline Azadirachtin & AzaGuard & $\mathrm{F}, \mathrm{G}, \mathrm{N}$ & Spray nymphs soon after egg hatch. \\
\hline $\begin{array}{l}\text { Beauveria bassiana } \\
\text { strain GHA }\end{array}$ & Mycotrol $\mathrm{O}^{3}$ & $\mathrm{~F}, \mathrm{G}, \mathrm{N}$ & $\begin{array}{l}\text { Typically it takes } 7-10 \text { days after the first spray to see control. See } \\
\text { label for precautions about use with fungicides. }\end{array}$ \\
\hline Carbaryl & Cutworm \& Cricket Bait & $\mathrm{F}, \mathrm{N}$ & Long, 7-day pre-harvest interval (PHI). \\
\hline Diazinon & $\begin{array}{l}\text { Diazinon } \mathrm{AG} 500^{4} \\
\text { Diazinon } 50 \mathrm{~W}^{4} \\
\text { Diazinon } \mathrm{AG} 600 \mathrm{WBC}^{4}\end{array}$ & $\mathrm{~F}, \mathrm{~N}$ & $\begin{array}{l}\text { Make a maximum of one foliar application per crop and a } \\
\text { maximum of one soil application per crop. Long, 5-day pre-harvest } \\
\text { interval (PHI). }\end{array}$ \\
\hline Pyrethrin & PyGanic EC $5.0^{3}$ & $\mathrm{~F}, \mathrm{G}, \mathrm{N}$ & \\
\hline \multicolumn{4}{|c|}{$\begin{array}{l}\text { Footnotes: } \\
1 \text { "F" indicates field production. "G" indicates greenhouse production. “ } \mathrm{N} \text { " indicates nursery production. } \\
{ }^{2} \text { Notes are taken from product labels and restrict use to the condition indicated (suppression, beet armyworm, exposed thrips, etc.), limit } \\
\text { number and patterns of applications, provide phytotoxicity precautions, etc. } \\
{ }^{3} \text { Product label indicates use in organic production. } \\
{ }^{4} \text { Product is a restricted-use pesticide. }\end{array}$} \\
\hline
\end{tabular}


Table 11. Pamera bug pesticidal control measures available for commercial strawberry production in Florida.

\begin{tabular}{|c|c|c|c|}
\hline Common Name & $\begin{array}{l}\text { Trade Name/ } \\
\text { Formulation }\end{array}$ & Production Site $^{1}$ & Notes from labels ${ }^{2}$ \\
\hline Azadirachtin & $\begin{array}{l}\text { Aza-Direct }^{3} \\
\text { Azatrol EC } \\
\text { Ecozin Plus 1.2\% } \mathrm{ME}^{3}\end{array}$ & $\mathrm{~F}, \mathrm{G}, \mathrm{N}$ & $\begin{array}{l}\text { True bugs; will not control adult insects. Reduces damage by } \\
\text { repelling and deterring feeding of all stages of insect. }\end{array}$ \\
\hline $\begin{array}{l}\text { Beauveria bassiana strain } \\
\text { GHA }\end{array}$ & $\begin{array}{l}\text { BotaniGard ES } \\
\text { Mycotrol O }\end{array}$ & $\mathrm{F}, \mathrm{G}, \mathrm{N}$ & $\begin{array}{l}\text { Typically it takes 7-10 days after the first spray to see control. See } \\
\text { labels for precautions about use with fungicides. }\end{array}$ \\
\hline Pyrethrin & $\begin{array}{l}\text { PyGanic Crop } \\
\text { Protection EC } 1.4^{3} \\
\text { PyGanic EC } 5.0^{3}\end{array}$ & $\mathrm{~F}, \mathrm{G}, \mathrm{N}$ & $\begin{array}{l}\text { Label does not list the insect but does not limit use to listed } \\
\text { insects. }\end{array}$ \\
\hline $\begin{array}{l}\text { Pyrethrin \& piperonyl } \\
\text { butoxide }\end{array}$ & $\begin{array}{l}\text { EverGreen EC 60-6 } \\
\text { Pyrenone Crop Spray }\end{array}$ & $\mathrm{F}, \mathrm{G}, \mathrm{N}$ & $\begin{array}{l}\text { Label does not list this insect but does not limit use to listed } \\
\text { insects. }\end{array}$ \\
\hline \multicolumn{4}{|c|}{$\begin{array}{l}\text { Footnotes: } \\
{ }_{1 \text { " } F " \text { indicates field production. “G" indicates greenhouse production. “N" indicates nursery production. }} \\
{ }^{2} \text { Notes are taken from product labels and restrict use to the condition indicated (suppression, beet armyworm, exposed thrips, etc.), limit } \\
\text { number and patterns of applications, provide phytotoxicity precautions, etc. } \\
{ }^{3} \text { Product label indicates use in organic production. }\end{array}$} \\
\hline
\end{tabular}


Table 12. Plant (Lygus) bug pesticidal control measures available for commercial strawberry production in Florida.

\begin{tabular}{|c|c|c|c|}
\hline Common Name & $\begin{array}{l}\text { Trade Name/ } \\
\text { Formulation }\end{array}$ & Production Site $^{1}$ & Notes from labels ${ }^{2}$ \\
\hline Acetamiprid & Assail 30SG & $\mathrm{F}, \mathrm{G}, \mathrm{N}$ & Do not make more than two applications per growing season. \\
\hline Azadirachtin & $\begin{array}{l}\text { Azatrol EC }{ }^{3} \\
\text { Neemix } 4.5^{3}\end{array}$ & $F, G, N$ & $\begin{array}{l}\text { Lygus bug; will not control adult insects. Reduces damage by repelling } \\
\text { and deterring feeding of all stages of insect. }\end{array}$ \\
\hline Beauveria bassiana & $\begin{array}{l}\text { Naturalis L } \\
\text { BotaniGard ES } \\
\text { Mycotrol O3 }\end{array}$ & $F, G, N$ & $\begin{array}{l}\text { Typically it takes } 7-10 \text { days after the first spray to see control. At least 3-5 } \\
\text { applications may be necessary before pests are adequately under control. } \\
\text { See labels for precautions about use with fungicides. }\end{array}$ \\
\hline Bifenthrin & Brigade WSB ${ }^{4}$ & $\mathrm{~F}, \mathrm{~N}$ & $\begin{array}{l}\text { Plant back restrictions: Plants for which bifenthrin tolerances exist may be } \\
\text { rotated at any time. All other crops may be rotated } 30 \text { days after the final } \\
\text { application of bifenthrin. }\end{array}$ \\
\hline $\begin{array}{l}\text { Bifenthrin \& } \\
\text { avermectin B1 }\end{array}$ & Athena ${ }^{4}$ & $\mathrm{~F}$ & $\begin{array}{l}\text { Lygus spp.; do not make more than two consecutive applications and four } \\
\text { applications per growing season. Plant back restrictions: For crops that } \\
\text { have bifenthrin and avermectin tolerances, the plant back is immediate. } \\
\text { All other crops may be rotated } 30 \text { days after the final application. }\end{array}$ \\
\hline $\begin{array}{l}\text { Bifenthrin \& } \\
\text { imidacloprid }\end{array}$ & Brigadier $^{4}$ & $\mathrm{~F}, \mathrm{~N}$ & $\begin{array}{l}\text { Lygus spp.; do not apply during or within } 10 \text { days after bloom or when } \\
\text { bees are actively foraging. Long, 7-day pre-harvest interval (PHI). Plant } \\
\text { back restrictions: Plants that have tolerances for both bifenthrin and } \\
\text { imidacloprid may be rotated at any time. Crops that have tolerances } \\
\text { for bifenthrin and not imidacloprid can be rotated } 12 \text { months after the } \\
\text { final application of Brigadier insecticide. Crops that have tolerances for } \\
\text { imidacloprid and not bifenthrin may be rotated } 30 \text { days after the final } \\
\text { application [label has list of crops]. }\end{array}$ \\
\hline Carbaryl & $\begin{array}{l}\text { Carbaryl 4L } \\
\text { Sevin Brand } 4 \mathrm{~F} \\
\text { Sevin Brand } 80 \mathrm{~S} \\
\text { Sevin Brand XLR Plus }\end{array}$ & $\mathrm{F}, \mathrm{N}$ & $\begin{array}{l}\text { Tarnished plant bug; do not plant rotational food and feed crops not listed } \\
\text { on this or other carbaryl labels in carbaryl-treated soil. Long, 7-day pre- } \\
\text { harvest interval (PHI). }\end{array}$ \\
\hline Etoxazole & Zeal & $\mathrm{F}, \mathrm{N}$ & $\begin{array}{l}\text { Do not make more than one Zeal application per growing season. For } \\
\text { lygus, spittlebug, tarnished plant bug or twospotted spider mite, use in } \\
\text { combination with Danitol 2.4EC. }\end{array}$ \\
\hline Fenpropathrin & Danitol 2.4EC 4 & $\mathrm{~F}, \mathrm{~N}$ & $\begin{array}{l}\text { Lygus, tarnished plant bug; do not exceed more than two applications } \\
\text { totaling } 2-2 / 3 \text { pts. ( } 0.8 \text { lbs. A.I.) per acre to the same planting in } 12 \\
\text { consecutive months. }\end{array}$ \\
\hline $\begin{array}{l}\text { Isaria fumosorosea } \\
\text { (formerly } \\
\text { Paecilomyces } \\
\text { fumosoroseus) }\end{array}$ & $\begin{array}{l}\text { Preferal } \\
\text { PFR-97 20\% WDG }{ }^{3}\end{array}$ & $\mathrm{~F}, \mathrm{G}, \mathrm{N}$ & $\begin{array}{l}\text { Most effective when relative humidity is } 80 \% \text { or higher for } 8-10 \text { hours. Can } \\
\text { be mixed with copper-based fungicides; do not mix with other fungicides } \\
\text { or apply within } 5 \text { days of fungicide applications other than copper. }\end{array}$ \\
\hline Malathion & $\begin{array}{l}\text { Gowan Malathion } 8 \mathrm{~F} \\
\text { Malathion 5EC }\end{array}$ & $\mathrm{F}, \mathrm{N}$ & \\
\hline Naled & Dibrom 8 Emulsive ${ }^{4}$ & $\mathrm{~F}, \mathrm{~N}$ & Do not apply when temperature is over $90^{\circ} \mathrm{F}$. \\
\hline Novaluron & Rimon 0.83EC & $\mathrm{F}, \mathrm{N}$ & Lygus; does not kill adult insects. \\
\hline $\begin{array}{l}\text { Oil (mineral, } \\
\text { paraffinic, } \\
\text { petroleum, } \\
\text { vegetable, etc.) }\end{array}$ & Ultra-fine Oil & $\mathrm{F}, \mathrm{N}$ & See label for phytotoxicity precautions. \\
\hline $\begin{array}{l}\text { Potassium salts of } \\
\text { fatty acids } \\
\text { (insecticidal soap) }\end{array}$ & DES-X $X^{3}$ & $\mathrm{~F}, \mathrm{~N}$ & Do not mix with sulfur. See label for phytotoxicity precautions. \\
\hline Pyrethrin & $\begin{array}{l}\text { PyGanic Crop } \\
\text { Protection EC } 1.4^{3} \\
\text { PyGanic EC } 5.0^{3}\end{array}$ & $\mathrm{~F}, \mathrm{G}, \mathrm{N}$ & \\
\hline $\begin{array}{l}\text { Pyrethrin \& } \\
\text { piperonyl butoxide }\end{array}$ & $\begin{array}{l}\text { EverGreen EC 60-6 } \\
\text { Pyrenone Crop Spray }\end{array}$ & $\mathrm{F}, \mathrm{G}, \mathrm{N}$ & \\
\hline $\begin{array}{l}\text { Sodium } \\
\text { tetraborohydrate } \\
\text { decahydrate }\end{array}$ & Prev-Am & $\mathrm{F}, \mathrm{N}$ & $\begin{array}{l}\text { Do not mix with chemicals containing sulfur or oils. Do not add adjuvants } \\
\text { to Prev-Am. }\end{array}$ \\
\hline
\end{tabular}




\begin{tabular}{|c|c|c|c|}
\hline Common Name & $\begin{array}{l}\text { Trade Name/ } \\
\text { Formulation }\end{array}$ & Production Site ${ }^{1}$ & Notes from labels ${ }^{2}$ \\
\hline Sorbitol octanoate & SorbiShield 90 & $\mathrm{~F}, \mathrm{G}, \mathrm{N}$ & A contact insecticide with limited residual activity. \\
\hline Thiamethoxam & Actara & $\mathrm{F}$ & Lygus bug suppression. \\
\hline $\begin{array}{l}\text { Thiamethoxam \& } \\
\text { chlorantraniliprole }\end{array}$ & Voliam Flexi & $\mathrm{F}$ & Lygus bug suppression. \\
\hline \multicolumn{4}{|c|}{$\begin{array}{l}\text { Footnotes: } \\
1 \text { "F" indicates field production. "G" indicates greenhouse production. "N" indicates nursery production. } \\
{ }^{2} \text { Notes are taken from product labels and restrict use to the condition indicated (suppression, beet armyworm, exposed thrips, etc.), limit } \\
\text { number and patterns of applications, provide phytotoxicity precautions, etc. } \\
{ }^{3} \text { Product label indicates use in organic production. } \\
{ }^{4} \text { Product is a restricted-use pesticide. }\end{array}$} \\
\hline
\end{tabular}


Table 13. Slug and Snail pesticidal control measures available for commercial strawberry production in Florida.

\begin{tabular}{|l|l|l|l|}
\hline Common Name & Trade Name/ Formulation & Production Site' & Notes from labels ${ }^{2}$ \\
\hline Iron phosphate & Sluggo-AG & F, G, N & \\
\hline Metaldehyde & $\begin{array}{l}\text { Durham Metaldehyde Granules } \\
\text { 3.5, 7.5 } \\
\text { OR-CAL Slug \& Snail Bait } \\
\text { Slug-Fest }\end{array}$ & F, N & \\
\hline $\begin{array}{l}\text { Footnotes: } \\
1 \text { "F" indicates field production. “G" indicates greenhouse production. "N" indicates nursery production. } \\
\text { 2 Notes are taken from product labels and restrict use to the condition indicated (suppression, beet armyworm, exposed thrips, etc.), limit } \\
\text { number and patterns of applications, provide phytotoxicity precautions, etc. }\end{array}$ \\
\hline
\end{tabular}


Table 14. Spider (widow spiders: Black widow and other widow spiders) pesticidal control measures available for commercial strawberry production in Florida.

\begin{tabular}{|c|c|c|c|}
\hline Common Name & $\begin{array}{l}\text { Trade Name/ } \\
\text { Formulation }\end{array}$ & Production Site ${ }^{1}$ & Notes from labels ${ }^{2}$ \\
\hline Bifenthrin $^{3}$ & Brigade WSB ${ }^{4}$ & $\mathrm{~F}, \mathrm{~N}$ & $\begin{array}{l}\text { Plant back restrictions: Plants for which bifenthrin tolerances exist } \\
\text { may be rotated at any time. All other crops may be rotated } 30 \\
\text { days after the final application of bifenthrin. }\end{array}$ \\
\hline Malathion $^{3}$ & $\begin{array}{l}\text { Ortho Malathion Plus } \\
\text { Insect Spray }\end{array}$ & $\mathrm{F}, \mathrm{N}$ & $\begin{array}{l}\text { Spiders; spot treatment to areas such as irrigation valves and } \\
\text { other equipment; apply as a course spray. }\end{array}$ \\
\hline Pyrethrin & $\begin{array}{l}\text { PyGanic Crop Protection } \\
\text { EC } 1.4^{5} \\
\text { PyGanic EC } 5.0^{5}\end{array}$ & $\mathrm{~F}, \mathrm{G}, \mathrm{N}$ & Spiders. \\
\hline $\begin{array}{l}\text { Pyrethrin \& piperonyl } \\
\text { butoxide }\end{array}$ & $\begin{array}{l}\text { EverGreen EC 60-6 } \\
\text { Pyrenone Crop Spray }\end{array}$ & $F, G, N$ & Spiders. \\
\hline \multicolumn{4}{|c|}{$\begin{array}{l}\text { Footnotes: } \\
1 \text { "F" indicates field production. "G" indicates greenhouse production. " } \mathrm{N} \text { " indicates nursery production. } \\
{ }^{2} \text { Notes are taken from product labels and restrict use to the condition indicated (suppression, beet armyworm, exposed thrips, etc.), limit } \\
\text { number and patterns of applications, provide phytotoxicity precautions, etc. } \\
{ }^{3} \text { Bifenthrin and malathion are labeled for spider control in outdoor ornamentals and turf, but no claim is made for spider control in strawberry } \\
\text { crops. } \\
{ }^{4} \text { Product is a restricted-use pesticide. } \\
{ }^{5} \text { Product label indicates use in organic production. }\end{array}$} \\
\hline
\end{tabular}


Table 15. Thrips pesticidal control measures available for commercial strawberry production in Florida.

\begin{tabular}{|c|c|c|c|}
\hline Common Name & $\begin{array}{l}\text { Trade Name/ } \\
\text { Formulation }\end{array}$ & $\begin{array}{l}\text { Production } \\
\text { Site }^{1}\end{array}$ & Notes from labels ${ }^{2}$ \\
\hline Abamectin & Temprano $^{3}$ & $\mathrm{~F}$ & Suppression \\
\hline Acetamiprid & Assail 30SG & $\mathrm{F}, \mathrm{G}, \mathrm{N}$ & Do not make more than two applications per growing season. \\
\hline Azadirachtin & $\begin{array}{l}\text { Aza-Direct }^{4} \\
\text { Azatin XL } \\
\text { Azatrol EC } \\
\text { Ecozin Plus } 1.2 \% \mathrm{ME}^{4} \\
\text { Neemix } 4.5^{4}\end{array}$ & $\mathrm{~F}, \mathrm{G}, \mathrm{N}$ & $\begin{array}{l}\text { Will not control adult insects. Effective on all larval stages and pupae. Reduces } \\
\text { damage by repelling and deterring feeding of all stages of insect. }\end{array}$ \\
\hline Beauveria bassiana & $\begin{array}{l}\text { Naturalis L } \\
\text { BotaniGard 22WP } \\
\text { BotaniGard ES } \\
\text { Mycotrol O4 }\end{array}$ & $\mathrm{F}, \mathrm{G}, \mathrm{N}$ & $\begin{array}{l}\text { Typically it takes } 7-10 \text { days after the first spray to see control. At least } 3-5 \\
\text { applications may be necessary before pests are adequately under control. See } \\
\text { labels for precautions about use with fungicides. }\end{array}$ \\
\hline $\begin{array}{l}\text { Edible fish oil \& sesame } \\
\text { oil }\end{array}$ & $\begin{array}{l}\text { Organocide 3-in-1 } \\
\text { Garden Spray }{ }^{4}\end{array}$ & $\mathrm{~F}, \mathrm{G}, \mathrm{N}$ & \\
\hline Fenpyroximate & Portal & $\mathrm{F}, \mathrm{N}$ & $\begin{array}{l}\text { Citrus thrips suppression; do not make more than two applications per } \\
\text { season. }\end{array}$ \\
\hline $\begin{array}{l}\text { Isaria fumosorosea } \\
\text { (formerly Paecilomyces } \\
\text { fumosoroseus) }\end{array}$ & $\begin{array}{l}\text { Preferal } \\
\text { PFR-97 20\% WDG }{ }^{4}\end{array}$ & $\mathrm{~F}, \mathrm{G}, \mathrm{N}$ & $\begin{array}{l}\text { Thrips pupae; most effective when relative humidity is } 80 \% \text { or higher for } 8-10 \\
\text { hours. Can be mixed with copper-based fungicides; do not mix with other } \\
\text { fungicides or apply within } 5 \text { days of fungicide applications other than copper. }\end{array}$ \\
\hline Malathion & $\begin{array}{l}\text { Gowan Malathion 8F } \\
\text { Malathion 5EC }\end{array}$ & $\mathrm{F}, \mathrm{N}$ & \\
\hline Naled & Dibrom 8 Emulsive 3 & $\mathrm{~F}, \mathrm{~N}$ & Do not apply when temperature is over $90^{\circ} \mathrm{F}$. \\
\hline Neem oil & Trilogy 4 & $\mathrm{~F}, \mathrm{~N}$ & $\begin{array}{l}\text { Suppression; avoid tank mixes with captan, sulfur or other chemically similar } \\
\text { products because unpredictable results or leaf burn may occur. }\end{array}$ \\
\hline Novaluron & Rimon 0.83EC & $\mathrm{F}, \mathrm{N}$ & Does not kill adult insects. \\
\hline $\begin{array}{l}\text { Oil (mineral, paraffinic, } \\
\text { petroleum, vegetable, } \\
\text { etc.) }\end{array}$ & Ultra-Fine Oil & $\mathrm{F}, \mathrm{N}$ & See label for phytotoxicity precautions. \\
\hline Pyrethrin & $\begin{array}{l}\text { PyGanic Crop } \\
\text { Protection EC } 1.4^{4} \\
\text { PyGanic EC } 5.0^{4}\end{array}$ & $\mathrm{~F}, \mathrm{G}, \mathrm{N}$ & \\
\hline $\begin{array}{l}\text { Pyrethrin \& piperonyl } \\
\text { butoxide }\end{array}$ & $\begin{array}{l}\text { EverGreen EC 60-6 } \\
\text { Pyreth-It } \\
\text { Pyrenone Crop Spray }\end{array}$ & $\mathrm{F}, \mathrm{G}, \mathrm{N}$ & \\
\hline $\begin{array}{l}\text { Pyrethrin, piperonyl } \\
\text { butoxide \& silicon } \\
\text { dioxide }\end{array}$ & $\begin{array}{l}\text { Diatect II } \\
\text { Multipurpose }\end{array}$ & $\mathrm{F}, \mathrm{G}, \mathrm{N}$ & \\
\hline $\begin{array}{l}\text { Pyrethrin, rotenone \& } \\
\text { associated resins }\end{array}$ & Pyrellin EC & $\mathrm{F}, \mathrm{G}, \mathrm{N}$ & \\
\hline Sorbitol octanoate & SorbiShield 90 & $\mathrm{~F}, \mathrm{G}, \mathrm{N}$ & A contact insecticide with limited residual activity. \\
\hline Spinetoram & Radiant SC & $\mathrm{F}, \mathrm{N}$ & $\begin{array}{l}\text { Resistance Management: Do not make more than two consecutive } \\
\text { applications of group } 5 \text { insecticides (spinetoram and spinosad). Do not make } \\
\text { more than five applications per calendar year. }\end{array}$ \\
\hline Spinosad & $\begin{array}{l}\text { Entrust }^{4} \\
\text { SpinTor 2SC }\end{array}$ & $\mathrm{F}, \mathrm{N}$ & $\begin{array}{l}\text { Resistance management: Rotate to a different class of insect control products } \\
\text { after two successive applications. Do not make more than five applications } \\
\text { per year. }\end{array}$ \\
\hline Steinernema feltiae & Nemasys & G & Western flower thrips. \\
\hline Sucrose octanoate & SucraShield & $\mathrm{F}, \mathrm{G}, \mathrm{N}$ & A contact insecticide with limited residual activity. \\
\hline \multicolumn{4}{|c|}{ 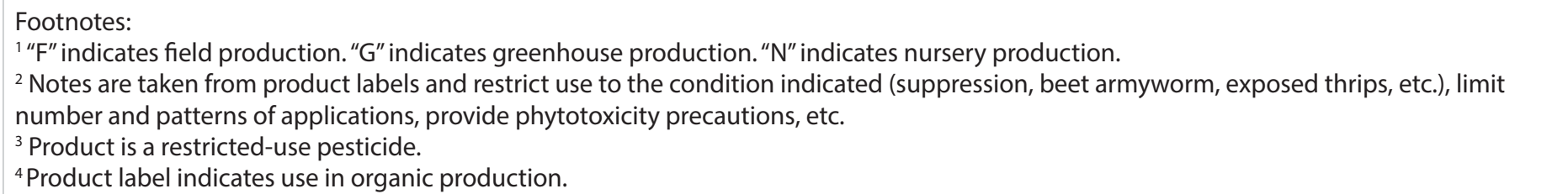 } \\
\hline
\end{tabular}

